\title{
The nonlinear Bernstein-Schrödinger equation in Economics
}

\author{
Alfred Galichon* $\quad$ Scott Duke Kominers ${ }^{\dagger} \quad$ Simon Weber ${ }^{\ddagger}$
}

July 17, 2018

In this note, we will review and extend some results from our previous work [8] where we introduced a novel approach to imperfectly transferable utility and unobserved heterogeneity in tastes, based on a nonlinear generalization of the Bernstein-Schrödinger equation. We consider an assignment problem where agents from two distinct populations may form pairs, which generates utility to each agent. Utility may be transfered across partners, possibly with frictions. This general framework hence encompasses both the classic Non-Tranferable Utility model (NTU) of Gale and Shapley [6], sometimes called the "stable marriage problem", where there exists no technology to allow transfers between matched partners; and the Transferable Utility (TU) model of Becker [1] and Shapley-Shubik [14, a.k.a. "optimal assignment problem," where utility (money) is additively transferable across partners.

If the NTU assumption seems natural for many markets (including school choices), TU models are more appropriate in most settings where there can be bargaining (labour and marriage markets for example). However, even in those markets, there can be transfer frictions. For example, in marriage markets, the transfers between partners might take the form of favor exchange (rather than cash), and the cost of a favor to one partner may not exactly equal the benefit to the other.

In [8], we thus developped a general Imperfectly Transferable Utility model with unobserved heterogeneity, which includes as special cases the classic fully- and non-transferable utility models, but also extends to collective models, and settings with taxes on transfers, deadweight losses, and risk aversion. As we argue in the present note, the models we consider in [8] obey a particularly simple system of equations we dubbed "Nonlinear Bernstein-Schrödinger equation". The present contribution present a general result for the latter equation, and also derives several consequences. The main result is derived in Section 1. Section 2 and Section 3 consider equilibrium assignment problems with and without heterogeneity. Finally, we provide a discussion of our results in Section 4 .

\footnotetext{
*Economics Department, Sciences Po, Paris, and New York University. Email: alfred.galichon@sciencespo.fr. Galichon gratefully acknowledges funding from the European Research Council under the European Union's Seventh Framework Programme (FP7/2007-2013) / ERC grant agreements no 313699 and 295298, and FiME.

${ }^{\dagger}$ Society of Fellows, Harvard University. Email: kominers@fas.harvard.edu. Kominers gratefully acknowledges the support of NSF grant CCF-1216095 and the Harvard Milton Fund.

${ }^{\ddagger}$ Economics Department, Sciences Po, Paris. Address: Sciences Po, Department of Economics, 27 rue SaintGuillaume, 75007 Paris, France. Email: simon.weber@sciencespo.fr.
} 


\section{The main result}

For $x \in \mathcal{X}$ and $y \in \mathcal{Y}$, we consider a function $M_{x y}: \mathbb{R}^{2} \rightarrow \mathbb{R}$, and $M_{x 0}: \mathbb{R} \rightarrow \mathbb{R}$ and $M_{0 y}: \mathbb{R} \rightarrow$ $\mathbb{R}$. Let $\left(n_{x}\right)_{x \in \mathcal{X}}$ and $\left(m_{y}\right)_{y \in \mathcal{Y}}$ be vectors of positive numbers. Consider the nonlinear BernsteinSchrödinger system, which consists in looking for two vectors $u \in \mathbb{R}^{\mathcal{X}}$ and $v \in \mathbb{R}^{\mathcal{Y}}$ such that

$$
\left\{\begin{array}{l}
M_{x 0}\left(u_{x}\right)+\sum_{y \in \mathcal{Y}} M_{x y}\left(u_{x}, v_{y}\right)=n_{x} \\
M_{0 y}\left(v_{y}\right)+\sum_{x \in \mathcal{X}} M_{x y}\left(u_{x}, v_{y}\right)=m_{y}
\end{array} .\right.
$$

Theorem 1. Assume $M_{x y}$ satisfies the following three conditions:

(i) Continuity. The maps $M_{x y}:\left(u_{x}, v_{y}\right) \longmapsto M_{x y}\left(u_{x}, v_{y}\right), M_{x 0}:\left(u_{x}\right) \longmapsto M_{x 0}\left(u_{x}\right)$ and $M_{0 y}$ : $\left(v_{y}\right) \longmapsto M_{0 y}\left(v_{y}\right)$ are continuous

(ii) Monotonicity. The map $M_{x y}:\left(u_{x}, v_{y}\right) \longmapsto M_{x y}\left(u_{x}, v_{y}\right)$ is monotonically decreasing, i.e if $u_{x} \leq u_{x}^{\prime}$ and $u_{y} \leq u_{y}^{\prime}$, then $M_{x y}\left(u_{x}, v_{y}\right) \geq M\left(u_{x}^{\prime}, v_{y}^{\prime}\right)$. The maps $M_{x 0}:\left(u_{x}\right) \longmapsto M_{x 0}\left(u_{x}\right)$ and $M_{0 y}:\left(v_{y}\right) \longmapsto M_{0 y}\left(v_{y}\right)$ are monotonically decreasing.

(iii) Limits. For each $v_{y}, \lim _{u_{x} \rightarrow \infty} M_{x y}\left(u_{x}, v_{y}\right)=0$ and $\lim _{u_{x} \rightarrow-\infty} M_{x y}\left(u_{x}, v_{y}\right)=+\infty$, and for each $u_{x}, \lim _{u_{y} \rightarrow \infty} M_{x y}\left(u_{x}, v_{y}\right)=0$ and $\lim _{u_{y} \rightarrow-\infty} M_{x y}\left(u_{x}, v_{y}\right)=+\infty$. Additionally, $\lim _{u_{x \rightarrow \infty}} M_{x 0}\left(u_{x}\right)=$ 0 and $\lim _{u_{y} \rightarrow \infty} M_{0 y}\left(u_{y}\right)=0$, and $\lim _{u_{x} \rightarrow-\infty} M_{x 0}\left(u_{x}\right)=+\infty$ and $\lim _{u_{y} \rightarrow-\infty} M_{0 y}\left(u_{y}\right)=+\infty$.

Then there exists a solution to the nonlinear Bernstein-Schrödinger system. Further, if the maps $M$ are $C^{1}$, the solution is unique.

This theorem appears in [8] under a slightly different form. The proof is interesting as it provides an algorithm of determination of $u$ and $v$. It is an important generalization of the Iterated Projection Fitting Procedure (see [5], [12, and [13]), which has been rediscovered and utilized many times under different names for various applied purposes: "RAS algorithm" [10, "biproportional fitting", "Sinkhorn Scaling" 4], etc. However, all these techniques and their variants can be recast as particular cases of the method described in the proof of Theorem 1 For convenience, we recall the algorithm used to provide a constructive proof of existence.

\section{Algorithm 1.}

Step $0 \mid$ Fix the initial value of $v_{y}$ at $v_{y}^{0}=+\infty$.

Step $2 t+1 \quad$ Keep the values $v_{y}^{2 t}$ fixed. For each $x \in \mathcal{X}$, solve for the value, $u_{x}^{2 t+1}$ such that equality $\sum_{y \in \mathcal{Y}} M_{x y}\left(u_{x}, v_{y}^{2 t}\right)+M_{x 0}\left(u_{x}\right)=n_{x}$ holds.

Step $2 t+2 \quad$ Keep the values $u_{x}^{2 t+1}$ fixed. For each $y \in \mathcal{Y}$, solve for which is the value, $v_{y}^{2 t+2}$ such that equality $\sum_{x \in \mathcal{X}} M_{x y}\left(u_{x}^{2 t+1}, v_{y}\right)+M_{0 y}\left(v_{y}\right)=m_{y}$ holds.

$u^{t}$ and $v^{t}$ converge monotonically to a solution of the Bernstein-Schrödinger system.

In practice a precision level $\epsilon>0$ will be chosen, and the algorithm will terminate when $\sup _{y}\left|v_{y}^{2 t+2}-v_{y}^{2 t}\right|<\epsilon$.

Proof. (i) Existence. The proof of existence is an application of Tarski's fixed point theorem and relies on the previous Algorithm. We need to prove that the construction of $u_{x}^{2 t+1}$ and $v_{y}^{2 t+2}$ at each step is well defined. Consider step $2 t+1$. For each $x \in \mathcal{X}$, the equation to solve is

$$
\sum_{y \in \mathcal{Y}} M_{x y}\left(u_{x}, v_{y}\right)+M_{x 0}\left(u_{x}\right)=n_{x}
$$

but the right handside is a continuous and decreasing function of $u_{x}$, tends to 0 when $u_{x} \rightarrow+\infty$ and tends to $+\infty$ when $u_{x} \rightarrow-\infty$. Note that by letting $v_{y} \rightarrow+\infty$, the terms in the sum tends 
to 0 , providing a lower bound for $u_{x}$. Hence $u_{x}^{2 t+1}$ is well defined and belongs in $\left(M_{x 0}^{-1}\left(n_{x}\right),+\infty\right)$, and let us denote

$$
u_{x}^{2 t+1}=F_{x}\left(v^{2 t}\right)
$$

and clearly, $F$ is anti-isotone, meaning that $v_{y}^{2 t} \leq \tilde{v}_{y}^{2 t}$ for all $y \in \mathcal{Y}$ implies $F_{x}\left(\tilde{v}^{2 t}\right) \leq F_{x}\left(v^{2 t}\right)$ for all $x \in \mathcal{X}$. By the same token, at step $2 t+2, v_{y}^{2 t+2}$ is well defined in $\left(M_{0 y}^{-1}\left(m_{y}\right),+\infty\right)$, and let us denote

$$
v_{y}^{2 t+2}=G_{y}\left(u^{2 t+1}\right)
$$

where, similarly, $G$ is anti-isotone. Thus

$$
v^{2 t+2}=G \circ F\left(v^{2 t}\right)
$$

where $G \circ F$ is isotone. But $v_{y}^{2}<\infty=v_{y}^{0}$ implies that $v_{.}^{2 t+2} \leq G \circ F\left(v^{2 t}\right)$. Hence $\left(v^{2 t+2}\right)_{t \in \mathbb{N}}$ is a decreasing sequence, bounded from below by 0 . As a result $v^{2 t+2}$ converges. Letting $\bar{v}$. its limit, and letting $\bar{u}=F(\bar{v})$, one can see that $(\bar{u}, \bar{v})$ is a solution to the nonlinear Bernstein-Schrödinger system. (ii) Unicity. Introduce map $\zeta$ defined by

$$
\zeta:\left(u_{x}, v_{y}\right) \rightarrow\left(\begin{array}{l}
\zeta_{x}=\sum_{y \in \mathcal{Y}} M_{x y}\left(u_{x}, v_{y}\right)+M_{x 0}\left(u_{x}\right) \\
\zeta_{y}=\sum_{x \in \mathcal{X}} M_{x y}\left(u_{x}, v_{y}\right)+M_{0 y}\left(v_{y}\right)
\end{array}\right)
$$

One has

$$
D \zeta\left(u_{x}, v_{y}\right)=\left(\begin{array}{l|l}
A & B \\
\hline C & D
\end{array}\right)
$$

where:

- $A=\left(\partial \zeta_{x} / \partial u_{x^{\prime}}\right)_{x x^{\prime}}=\sum_{y^{\prime} \in \mathcal{Y}} \partial_{u_{x}} M_{x y^{\prime}}\left(u_{x}, v_{y^{\prime}}\right)+1$ if $x=x^{\prime}, 0$ otherwise.

- $B=\left(\partial \zeta_{x} / \partial v_{y}\right)_{x y}=\partial_{v_{y}} M_{x y}\left(u_{x}, v_{y}\right)$

- $C=\left(\partial \zeta_{y} / \partial u_{x}\right)_{y x}=\partial_{u_{x}} M_{x y}\left(u_{x}, v_{y}\right)$

- $D=\left(\partial \zeta_{y} / \partial v_{y^{\prime}}\right)_{y y^{\prime}}=\sum_{x^{\prime} \in \mathcal{X}} \partial_{v_{y}} M_{x^{\prime} y}\left(u_{x^{\prime}} v_{y}\right)+1$ if $y=y^{\prime}, 0$ otherwise.

It is straightforward to show that the matrix $D \zeta$ is dominant diagonal. A result from [11] states that a dominant diagonal matrix with positive diagonal entries is a $\mathrm{P}$-matrix. Hence $D \zeta\left(u_{x}, v_{y}\right)$ is a P-matrix. Applying Theorem 4 in [9] it follows that $\zeta$ is injective.

\section{Equilibrium Assignment Problem}

In this section, we consider the equilibrium assignment problem, which is a far-reaching generalization of the optimal assignment problem. To describe this framework, consider two finite populations $\mathcal{I}$ and $\mathcal{J}$, and a two-sided matching framework (for simplicity, we will call "men" and "women" the two sides of this market) with imperfect transfers and without heterogeneity. Agents $i \in \mathcal{I}$ and $j \in \mathcal{J}$ get respectively utility $u_{i}$ and $v_{j}$ they get at equiliburium. If $i$ or $j$ remains unmatched, they get utility 0 ; however, if they match together, they may get any respective utilities $u_{i}$ and $v_{j}$ such that the feasibility constraint is imposed

$$
\Psi_{i j}\left(u_{i}, v_{j}\right) \leq 0,
$$

where the transfer function $\Psi_{i j}$ is assumed to be continuous and isotone with respect to its arguments. Note that at equilibrium, $u_{i} \geq 0$ and $v_{j} \geq 0$ as the agents always have the option to 
remain unassigned; by the same token, if for any pair $i, j$ (matched or not), one cannot have a strict inequality in (1), otherwise $i$ and $j$ would have an incentive to form a blocking pair, and achieve a higher payoff than their equilibrium payoff. Thus the stability condition $\Psi_{i j}\left(u_{i}, v_{j}\right) \geq 0$, holds in general. Let $\mu_{i j}=1$ if $i$ and $j$ are matched, and 0 otherwise; we have therefore that $\mu_{i j}>0$ implies that $\Psi_{i j}\left(u_{i}, v_{j}\right)=0$. This allows us to define an equilibrium outcome.

Definition 1. The equilibrium assignment problem defined by $\Psi$ has an equilibrium outcome $\left(\mu_{i j}, u_{i}, v_{j}\right)$ whenever the following conditions are met: (i) $\mu_{i j} \geq 0, u_{i} \geq 0$ and $v_{j} \geq 0$ (ii) $\sum_{j} \mu_{i j} \leq 1$ and $\sum_{i} \mu_{i j} \leq 1$ (iii) $\Psi_{i j}\left(u_{i}, v_{j}\right) \geq 0$ (iv) $\mu_{i j}>0$ implies $\Psi_{i j}\left(u_{i}, v_{j}\right)=0$.

Note that, by the Birkhoff-von Neumann theorem, the existence of an equilibrium in this problem leads to the existence of an equilibrium satisfying the stronger integrality requirement $\mu_{i j} \in\{0,1\}$. This general framework allow us to express the optimal assignment problem (matching with Transferable Utility), as the case where

$$
\Psi_{i j}\left(u_{i}, v_{j}\right)=u_{i}+v_{j}-\Phi_{i j}
$$

while in the NTU case

$$
\Psi_{i j}\left(u_{i}, v_{j}\right)=\max \left(u_{i}-\alpha_{i j}, v_{j}-\gamma_{i j}\right) .
$$

Other interesting cases are considered in [8]. For instance, the Linearly Transferable Utility (LTU) model, where

$$
\Psi_{i j}\left(u_{i}, v_{j}\right)=\lambda_{i j}\left(u_{i}-\alpha_{i j}\right)+\zeta_{i j}\left(v_{j}-\gamma_{i j}\right)
$$

with $\lambda_{i j}, \zeta_{i j}>0$, and the Exponentially Transferable Utility $(E T U)$ model, in which $\Psi_{i j}$ takes the form

$$
\Psi_{i j}\left(u_{i}, v_{j}\right)=\tau \log \left(\frac{\exp \left(u_{i} / \tau\right)+\exp \left(v_{j} / \tau\right)}{2}\right) .
$$

In the ETU model, the parameter $\tau_{i j}$ is defined as the degree of transferability, since $\tau \rightarrow+\infty$ recovers the TU case and $\tau \rightarrow 0$ recovers the NTU framework.

Theorem 2. Assume $\Psi$ is such that: (a) For any $x \in \mathcal{X}$ and $y \in \mathcal{Y}$, we have $\Psi_{x y}(\cdot, \cdot)$ continuous. (b) For any $x \in \mathcal{X}, y \in \mathcal{Y}, t \leq t^{\prime}$ and $r \leq r^{\prime}$, we have $\Psi_{x y}(t, r) \leq \Psi_{x y}\left(t^{\prime}, r^{\prime}\right)$; furthermore, when $t<t^{\prime}$ and $r<r^{\prime}$, we have $\Psi_{x y}(t, r)<\Psi_{x y}\left(t^{\prime}, r^{\prime}\right)$. (c) For any sequence $\left(t_{n}, r_{n}\right)$, if $\left(r_{n}\right)$ is bounded and $t_{n} \rightarrow+\infty$, then $\lim \inf \Psi_{x y}\left(t_{n}, r_{n}\right)>0$. Analogously, if $\left(t_{n}\right)$ is bounded and $r_{n} \rightarrow+\infty$, then $\liminf \Psi_{x y}\left(t_{n}, r_{n}\right)>0$. (d) For any sequence $\left(t_{n}, r_{n}\right)$ such that if $\left(t_{n}-r_{n}\right)$ is bounded and $t_{n} \rightarrow-\infty$ (or equivalently, $\left.r_{n} \rightarrow+\infty\right)$, we have that $\lim \sup \Psi_{x y}\left(t_{n}, r_{n}\right)<0$. Then the equilibrium assignment problem defined by $\Psi_{i j}$ has an equilibrium outcome.

Proof. Consider $T>0$ and let

$$
\begin{aligned}
M_{i j}\left(u_{i}, v_{j}\right) & =\exp \left(-\frac{\Psi_{i j}\left(u_{i}, v_{j}\right)}{T}\right) \\
M_{i 0}\left(u_{i}\right) & =\exp \left(-\frac{u_{i}}{T}\right) \\
M_{0 j}\left(v_{j}\right) & =\exp \left(-\frac{v_{j}}{T}\right)
\end{aligned}
$$

and consider the Bernstein-Schrödinger system

$$
\left\{\begin{array}{c}
M_{i 0}\left(u_{i}\right)+\sum_{j \in \mathcal{J}} M_{i j}\left(u_{i}, v_{j}\right)=1 \\
M_{0 j}\left(v_{j}\right)+\sum_{i \in \mathcal{I}} M_{i j}\left(u_{i}, v_{j}\right)=1
\end{array} .\right.
$$


We need to show that $M_{x y}(.,),. M_{x 0}($.$) and M_{0 y}($.$) satisfy the properties stated in Theorem 1, It is$ straightforward to show that conditions (i) and (ii) in Theorem 1 follow directly from assumptions (a) and (b) on $\Psi$. Moreover, letting $T \rightarrow 0^{+}$, assumptions (c) and (d) together imply that condition (iii) is satisfied. Hence we can apply Theorem 1, and it follows that a solution $u_{i}^{T}, v_{j}^{T}$ to the system exists. Note that $M_{i 0}\left(u_{i}^{T}\right) \leq 1$ and $M_{0 j}\left(v_{j}^{T}\right) \leq 1$ imply that $u_{i}^{T} \geq 0$ and $v_{j}^{T} \geq 0$. Now, consider the sequence obtained by taking $T=k, k \in \mathbb{N}$. Then, up to a subsequence extraction, we may assume $u_{i}^{k} \rightarrow \bar{u}_{i} \in \mathbb{R}^{+} \cup\{+\infty\}$ and $v_{j}^{k} \rightarrow \bar{v}_{j} \in \mathbb{R}^{+} \cup\{+\infty\}$. It follows that $\Psi_{i j}\left(u_{i}^{k}, v_{j}^{k}\right)$ converges in $\mathbb{R}^{+} \cup\{+\infty\}$, hence $\mu_{i j}^{k}=M_{i j}\left(u_{i}^{k}, v_{j}^{k}\right)$ converges toward $\bar{\mu}_{i j} \in[0,1]$. Similarly, the limits $\bar{\mu}_{i 0}$ and $\bar{\mu}_{0 j}$ exist in $[0,1]$. Hence (i) in Definition 1 is met. By continuity, $\bar{\mu}$ satisfies

$$
\left\{\begin{array}{c}
\bar{\mu}_{i 0}+\sum_{j \in \mathcal{J}} \bar{\mu}_{i j}=1 \\
\bar{\mu}_{0 j}+\sum_{i \in \mathcal{I}} \bar{\mu}_{i j}=1
\end{array},\right.
$$

which established (ii). Let us show that (iii) holds, that is, that $\Psi_{i j}\left(u_{i}, v_{j}\right) \geq 0$ for any $i$ and $j$. Assume otherwise. Then there exists $\epsilon>0$ such that for $k$ large enough, $\Psi_{i j}\left(u_{i}^{k}, v_{j}^{k}\right)<-\epsilon$, so that $\mu_{i j}^{k}>\exp (\epsilon / T) \rightarrow+\infty$, contradicting $\bar{\mu}_{i j} \leq 1$. Thus, we have established (iii). Finally, we show that (iv) holds. Assume otherwise. Then there is $i$ and $j$ such that $\bar{\mu}_{i j}>0$ and $\Psi_{i j}\left(\bar{u}_{i}, \bar{v}_{j}\right)>0$. This implies that there exists $\epsilon>0$ such that for $k$ large enough, $\mu_{i j}^{k}>\epsilon$, thus $\Psi_{i j}\left(u_{i}^{k}, v_{j}^{k}\right)<$ $-T \log \epsilon \rightarrow 0$, hence $\Psi_{i j}\left(\bar{u}_{i}, \bar{v}_{j}\right) \leq 0$, a contradiction. Hence (iv) holds; this completes the proof and establishes that $(\bar{\mu}, \bar{u}, \bar{v})$ is an equilibrium assignment.

\section{ITU matching with heterogeneity}

Following [8], we now assume that individuals may be gathered into groups of agents of similar observable characteristics, or types, but heterogeneous tastes. We let $\mathcal{X}$ and $\mathcal{Y}$ be the sets of types of men and women. An individual man $i \in \mathcal{I}$ has type $x_{i} \in \mathcal{X}$; similarly, an individual woman $j \in \mathcal{J}$ has type $y_{j} \in \mathcal{Y}$. We assume that there is a mass $n_{x}$ of men of type $x$ and $m_{y}$ of women of type $y$, respectively. Assume further that

$$
\Psi_{i j}\left(u_{i}, v_{j}\right)=\Psi_{x_{i} y_{j}}\left(u_{i}-T \varepsilon_{i y}, v_{j}-T \eta_{x j}\right),
$$

where $\epsilon$ and $\eta$ are i.i.d. random vectors drawn from a Gumbel distribution, and where $T>0$ is a temperature parameter. Unassigned agents get $T \varepsilon_{i 0}$ and $T \eta_{0 j}$. For all $i$ such that $x_{i}=x$ and $y_{j}=y$, the stability condition implies

$$
\Psi_{x_{i} y_{j}}\left(u_{i}-T \varepsilon_{i y}, v_{j}-T \eta_{x j}\right) \geq 0 .
$$

Hence,

$$
\min _{\substack{i: x_{i}=x \\ j: y_{j}=y}} \Psi_{x_{i} y_{j}}\left(u_{i}-T \varepsilon_{i y}, v_{j}-T \eta_{x j}\right) \geq 0
$$

Thus, letting

$$
U_{x y}=\min _{i: x_{i}=x}\left\{u_{i}-T \varepsilon_{i y}\right\} \text { and } V_{x y}=\min _{j: y_{j}=y}\left\{v_{j}-T \eta_{x j}\right\},
$$

we have $\Psi_{x y}\left(U_{x y}, V_{x y}\right) \geq 0$, and with

$$
\mu_{x y}=\sum_{\substack{i: x_{i}=x \\ j: y_{j}=y}} \mu_{i j}
$$


we have that $\mu_{x y}>0$ implies $\Psi_{x y}\left(U_{x y}, V_{x y}\right)=0$, and by a standard argument (the random vectors $\epsilon$ and $\eta$ are drawn from distributions with full support, hence there will be at least a man $i$ of type $x$ and a woman $j$ of type $y$ such that $i$ prefers type $y$ and $j$ prefers type $x$, that is, $\mu_{x y}>0$ for all $x$ and $y$ )

$$
\Psi_{x y}\left(U_{x y}, V_{x y}\right)=0 \forall x \in \mathcal{X}, y \in \mathcal{Y} .
$$

Note that this is an extension to the ITU case of the analysis in Galichon and Salanié [7], building on Choo and Siow [3]. We have that

$$
u_{i}=\max _{y}\left\{U_{x y}+T \varepsilon_{i y}, T \varepsilon_{i 0}\right\} \text { and } v_{j}=\max _{x}\left\{V_{x y}+T \eta_{x j}, T \eta_{0 j}\right\}
$$

thus a standard result from Extreme Value Theory (see Choo and Siow [3] for a derivation) yields

$$
U_{x y}=T \log \frac{\mu_{x y}}{\mu_{x 0}} \text { and } V_{x y}=T \log \frac{\mu_{x y}}{\mu_{0 y}},
$$

so we see that $\mu_{x y}$ satisfies

$$
\left\{\begin{array}{c}
\mu_{x 0}+\sum_{y \in \mathcal{Y}} \mu_{x y}=n_{x} \\
\mu_{0 y}+\sum_{x \in \mathcal{X}} \mu_{x y}=m_{y} \\
\Psi_{x y}\left(T \log \frac{\mu_{x y}}{\mu_{x 0}}, T \log \frac{\mu_{x y}}{\mu_{0 y}}\right)=0
\end{array} .\right.
$$

The various cases of interest discussed above, namely TU, NTU, LTU, and ETU cases yield, respectively,

$$
\begin{aligned}
\mu_{x y} & =\mu_{x 0}^{1 / 2} \mu_{0 y}^{1 / 2} \exp \frac{\Phi_{x y}}{2}(\mathrm{TU}) \\
\mu_{x y} & =\min \left(\mu_{x 0} e^{\alpha_{x y}}, \mu_{0 y} e^{\gamma_{x y}}\right)(\mathrm{NTU}) \\
\mu_{x y} & =e^{\left(\lambda_{x y} \alpha_{x y}+\zeta_{x y} \gamma_{x y}\right) /\left(\lambda_{x y}+\zeta_{x y}\right)} \mu_{x 0}^{\lambda_{x y} /\left(\lambda_{x y}+\zeta_{x y}\right)} \mu_{0 y}^{\zeta_{x y} /\left(\lambda_{x y}+\zeta_{x y}\right)} \\
\mu_{x y} & =\left(\frac{e^{-\alpha_{x y} / \tau_{x y}} \mu_{x 0}^{-1 / \tau_{x y}}+e^{-\gamma_{x y} / \tau_{x y}} \mu_{0 y}^{-1 / \tau_{x y}}}{2}\right)^{-\tau_{x y}} \quad(\mathrm{ETU}),
\end{aligned}
$$

(see [3]). To apply Theorem 1, we let $M_{x y}\left(u_{x}, v_{y}\right)$ be the value $m$ solution to (for a proof of existence and uniqueness of such a solution, see Lemma 1 of [8])

$$
\Psi_{x y}\left(T \log m+u_{x}, T \log m+v_{y}\right)=0,
$$

and let

$$
M_{x 0}\left(u_{x}\right)=\exp \left(\frac{-u_{x}}{T}\right) \text { and } M_{0 y}\left(v_{y}\right)=\exp \left(\frac{-v_{y}}{T}\right) .
$$

In [8], we rewrote system (2) as a nonlinear Bernstein-Schrödinger system, namely

$$
\left\{\begin{array}{l}
M_{x 0}\left(u_{x}\right)+\sum_{y \in \mathcal{Y}} M_{x y}\left(u_{x}, v_{y}\right)=n_{x} \\
M_{0 y}\left(v_{y}\right)+\sum_{x \in \mathcal{X}} M_{x y}\left(u_{x}, v_{y}\right)=m_{y}
\end{array} .\right.
$$

Theorem 3. The nonlinear Bernstein-Schrödinger system in (2) has a unique solution

Proof. The proof follows directly from the application of Theorem 1. It is easy to check that the conditions on $M_{x 0}($.$) and M_{0 y}($.$) required by Theorem 1$ are met in this case. Lemma 1 in [8] provides a proof that $M_{x y}$ satisfies these conditions. 


\section{Discussion}

In this note, we have argued how matching problems may be formulated as a system of nonlinear equations, also known as the Bernstein-Schrödinger equation or Schrödinger's problem [15. We have shown existence and uniqueness of a solution under certain conditions, and have explicited the link with various matching problems, with or without heterogeneity. Solving such a system of equations requires an algorithm that we call the Iterative Projection Fitting Procedure (IPFP); in practice, this algorithm converges very quickly. Our setting can be extended in several ways. One of them is to consider the case with unassigned agents. In that case, we have the additional constraint that $\sum_{x} n_{x}=\sum_{y} m_{y}$, thus the nonlinear Bernstein-Schrödinger system, which in this case writes as

$$
\left\{\begin{array}{l}
\sum_{y \in \mathcal{Y}} M_{x y}\left(u_{x}, v_{y}\right)=n_{x} \\
\sum_{x \in \mathcal{X}} M_{x y}\left(u_{x}, v_{y}\right)=m_{y}
\end{array}\right.
$$

has a degree of freedom, as the sum over $x \in \mathcal{X}$ of the first set of equations coincides with the sum over $y \in \mathcal{Y}$ of the second one. The one-dimensional manifold of solutions of this problem is studied in [2].

\section{References}

[1] Becker, G. S. (1973): "A Theory of Marriage: Part I," Journal of Political Economy, 81(4), pp. 813-46.

[2] Carlier, G., and Galichon, A. (2014): "A remark on the manifold of solutions of a nonlinear Schrödinger system," Working Paper.

[3] Choo, E., and A. Siow (2006): "Who Marries Whom and Why," Journal of Political Economy, 114(1), pp. 175-201.

[4] Cuturi, M. (2013): "Sinkhorn Distances: Lightspeed Computation of Optimal Transport." Advances in Neural Information Processing Systems, pp. 2292-2300.

[5] Deming, W. E., and Stephan, F. F. (1940): "On a Least Squares Adjustment of a Sampled Frequency Table When the Expected Marginal Totals are Known," Annals of Mathematical Statistics, 11(4), pp. 427-444.

[6] Gale, D., \& Shapley, L. S. (1962): "College Admissions and the Stability of Marriage," American Mathematical Monthly, 69(1), pp. 9-15.

[7] Galichon, A., and B. Salanié (2014): "Cupid's Invisible Hand: Social Surplus and Identification in Matching Models," Working paper.

[8] Galichon, A., S. D. Kominers, and S. Weber (2014): "An Empirical Framework for Matching with Imperfectly Transferable Utility," Working paper.

[9] Gale, D., and Nikaido, H. (1965): "The Jacobian matrix and global univalence of mappings," Mathematische Annalen, 159(2), pp. 81-93.

[10] Kruithof, J. (1937): "Telefoonverkeersrekening," De Ingenieur, 52, E15-E25.

[11] McKenzie, L. W. (1959): Matrices with dominant diagonals and economic theory. Mathematical Methods in the Social Sciences 1959. Stanford Univ. Press 1960. 
[12] Ruschendorf, L. (1995): "Convergence of the Iterative Proportional Fitting Procedure," Annals of Statistics, 23(4), pp. 1160-1174.

[13] Rüschendorf, L., and Thomsen, W. (1998): "Closedness of Sum Spaces andthe Generalized Schrödinger Problem," Theory of Probability $\&$ Its Applications, 42(3), pp. 483-494.

[14] Shapley, L., and M. Shubik (1972): "The Assignment Game I: The Core," International Journal of Game Theory, 1, pp. 111-130.

[15] Schrödinger, E. (1931): "Über die Umkehrung der Naturgesetze. Sitzungsberichte Preuss," Akad. Wiss. Berlin, Phys. Math., 144, pp. 144-153. 